\title{
Photonic mode dispersion of a two-dimensional distributed feedback polymer laser
}

\author{
G. A. Turnbull,${ }^{1}$ P. Andrew, ${ }^{2}$ W. L. Barnes, ${ }^{2, *}$ and I. D. W. Samuel ${ }^{1, \dagger}$ \\ ${ }^{1}$ Organic Semiconductor Centre and Ultrafast Photonics Collaboration, School of Physics and Astronomy, \\ University of St. Andrews, St. Andrews, Fife, KY16 9SS, United Kingdom \\ ${ }^{2}$ Thin Film Photonics Group, School of Physics, University of Exeter, Stocker Road, Exeter, EX4 4QL, United Kingdom \\ (Received 7 October 2002; published 24 April 2003)
}

\begin{abstract}
We present an analysis of the photonic mode dispersion of a two-dimensional (2D) distributed feedback polymer laser based on the conjugated polymer poly[2-methoxy-5-(2'-ethylhexyloxy)-1,4-phenylene vinylene]. We use a combination of a simple model, together with experimental measurements of the photonic mode dispersion in transmission and emission, to explain the operating characteristics of the laser. The laser was found to oscillate at $636 \mathrm{~nm}$ on one edge of a photonic stop band in the photonic dispersion. A 2D coupling of modes traveling perpendicular to the orthogonal gratings was found to lead to a low divergence laser emission normal to the waveguide. At pump energies well above the oscillation threshold for this mode, a divergent, cross-shaped far-field emission was observed, resulting from a distributed feedback occurring over a wide range of wave vectors in one band of the photonic dispersion.
\end{abstract}

DOI: 10.1103/PhysRevB.67.165107 PACS number(s): 42.55.-f, 42.70.Jk, 42.70.Qs, 42.70.Mp

\section{INTRODUCTION}

In recent years, organic semiconductors have received considerable interest as novel laser gain media. ${ }^{1-25}$ Various materials have been studied, including conjugated polymers $^{1-17}$ and small semiconducting molecules doped with laser dyes. ${ }^{18-25}$ Such materials can exhibit strong optical gain over broad spectral ranges throughout the visible, and so are well suited for use in tuneable lasers ${ }^{9,11,22}$ or broadband amplifiers. ${ }^{17}$ To date, work in this area has involved only optically pumped lasers, although the prospect of a plastic diode laser simply fabricated from solution remains a much sought-after goal. ${ }^{4,5,20}$

An important challenge in the physics relevant to this goal involves understanding how photonic microstructures influence light emission from organic semiconductors. The simple processing of organics allows them to be easily fashioned into complex wavelength-scale structures. These can modify the local photonic environment of the semiconductor, which in turn affects the emission. Such structures can, for example, be used to provide resonant feedback in organic semiconductor lasers. ${ }^{4,5}$ Of various resonator geometries considered, distributed feedback (DFB) structures are among the most promising for the low-threshold operation required for electrical excitation. ${ }^{4,5} \mathrm{~A}$ number of DFB lasers have been demonstrated in organic semiconductors, employing single gratings, ${ }^{6-13,19-21}$ crossed double gratings, ${ }^{14,22}$ circular gratings, ${ }^{15,16}$ and photonic crystal structures. ${ }^{23-25}$ Through active control of the photonic modes it should be possible to control lasing properties such as threshold and output coupling. It is therefore important to understand the physics of how the local photonic environment modifies emission.

In this paper, we explore the problem of how the photonic dispersion controls emission from a two-dimensional (2D) DFB polymer laser, based on a crossed double-grating structure. We use direct measurements of the photonic band structure to explain the spectral and spatial emission features. Such measurements can reveal detailed structure of the photonic dispersion, without the need for complicated theoretical modeling. Importantly these measurements probe the actual sample under study, and not just a theoretical approximation to the structure. So by comparing the angle-dependent transmission and emission characteristics of the structure, we can gain important insight into mechanisms that influence the spectral and spatial properties of the laser emission. Specifically, we present a study of the operating characteristics of a laser based on the conjugated polymer poly(2-methoxy-5(2'-ethylhexyloxy)-1,4-phenylene vinylene) (MEH-PPV). We describe its emission properties when operating both below and above lasing threshold, and relate these properties to the photonic mode structure of the device. Such a comparison was previously found helpful in understanding the frequency selection mechanisms in 1D DFB polymer lasers. ${ }^{10,12}$ In the present work we make use of this approach to understand the more complex underlying physics of a $2 \mathrm{D}$ structure.

\section{LASER FABRICATION AND EXPERIMENTAL PROCEDURE}

The structure of the polymer DFB laser studied is shown schematically in Fig. 1(a). It comprised a crossed doublegrating corrugated silica substrate covered by a thin film of the conjugated polymer MEH-PPV. The silica-polymer-air structure formed an asymmetric slab waveguide that supported only the fundamental transverse electric mode for the wavelengths of interest.

The two-dimensional substrate corrugations were initially defined holographically in a photoresist layer on the silica substrate. Subsequent chemical development of the photoresist, and reactive-ion etching of the corrugation into the silica substrate, formed the 2D square array grating structure shown in the atomic force microscope (AFM) image in Fig. 1(b). The grating had a period of $409.1 \mathrm{~nm} \pm 0.1 \mathrm{~nm}$ in both directions, and a peak to dip height of $105 \mathrm{~nm} \pm 5 \mathrm{~nm}$. The grating was anharmonic, with a second-harmonic amplitude of approximately $10 \%$ of the fundamental amplitude. The corrugations in the structure provided both distributed feed- 
(a)

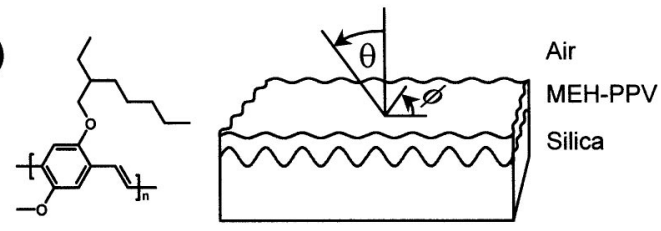

(b)

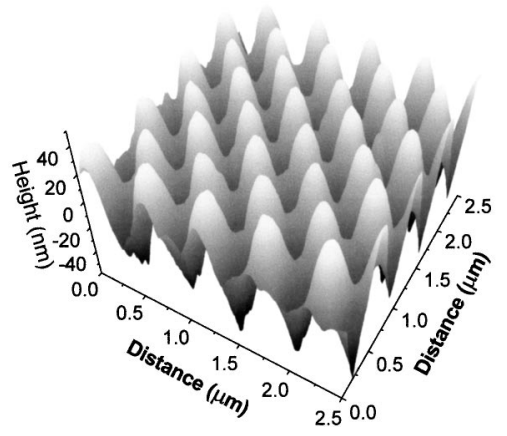

FIG. 1. (a) Waveguide structure of DFB laser and chemical structure of MEH-PPV. (b) Atomic force microscope image of the surface structure of the silica substrate.

back and output coupling of the guided optical mode via second- and first-order Bragg scattering, respectively.

To complete the structure, a thin film of MEH-PPV was spin coated from solution in chlorobenzene (5 mg MEH-PPV in $1 \mathrm{ml}$ chlorobenzene; spin speed of $1200 \mathrm{rpm}$ ) onto the silica. This formed a polymer layer with an average thickness of $\sim 100 \mathrm{~nm}$ as measured by a DekTak-3 surface profiler. As a result of the deposition process, the top surface of the polymer layer is substantially more planar than the silica substrate.

Immediately after fabrication, the sample was transferred to a vacuum chamber in which it was held under a dynamic vacuum of $\sim 10^{-4}$ mbar during the subsequent optical characterization. For the lasing measurements, the sample was excited at $500 \mathrm{~nm}$ by a dye laser that generated pulses of 500 ps duration, at $10-\mathrm{Hz}$ repetition rate. The dye laser output was attenuated using neutral density filters to energies below $100 \mathrm{~nJ}$, and then focused onto the sample to a circular spot of $\sim 200 \mu \mathrm{m}$. Output energies from the laser were measured using a calibrated silicon energy meter for a range of excitation powers. The spectral output from the laser was measured around normal incidence using a fiber-coupled chargecoupled device (CCD) spectrometer. The divergence of the laser output beam was measured using a scanning knife-edge technique at a distance of $120 \mathrm{~mm}$ from the front of the sample.

The angular emission pattern of photoluminescence from the structure was measured at excitation densities well below lasing threshold. Emission was measured at polar angles $\theta$ of between $0^{\circ}$ and $50^{\circ}$ to the waveguide normal, in the $0^{\circ}$ azimuthal plane (i.e., measurements in a plane parallel to the grooves of one of the gratings; see Fig. 1). For these measurements, the sample was excited at $532 \mathrm{~nm}$ using the second harmonic output of a continuous-wave diode-pumped Nd:YAG laser. The power of the pump laser was attenuated to a power of less than $1 \mathrm{~mW}$, and the beam focused to a circular spot of $\sim 100 \mu \mathrm{m}$ radius on the polymer surface. Polarized photoluminescence spectra were detected using the fiber-coupled CCD spectrometer for a range of polar angles around the normal to the waveguide plane. For these angular measurements, the 1-mm-diameter fiber was held at a distance of $50 \mathrm{~mm}$ from the excited region of the sample, thereby collecting an angular spread of the emission of $\sim 1^{\circ}$. The spectral resolution of the spectrometer was approximately $1.5 \mathrm{~nm}$ for the measurements shown in Figs. 8(a) and (c), and $0.3 \mathrm{~nm}$ for the measurements shown in Figs. 2 and 8(b) and (d).

The photonic band structure of the device was subsequently explored by measuring the transmission of light through the device using apparatus described in detail elsewhere. ${ }^{26}$ The sample was mounted on a rotation stage and illuminated with the spectrally filtered and collimated beam of a tungsten halogen white light lamp. A polarizer placed between the lamp and the sample was used to discriminate between the excitation of waveguide modes by TE and TM polarized light. The photonic mode dispersion of the sample could then be mapped out by measuring the absolute transmission as a function of angle and wavelength. Where the light couples into waveguide modes there is a reduction in transmission whose magnitude corresponds to the coupling strength between the incident radiation and the mode. The transmission was measured by monitoring the intensity of the signal and reference beams with photomultiplier tubes as the incident angle and wavelength were synchronously varied under computer control to acquire data equally spaced in both energy, $1 / \lambda$, and wave vector, $(\sin \theta) / \lambda$. The spectral resolution of the system was $\sim 1.5 \mathrm{~nm}$, limited by the spectral half width of the monochromator and the angular resolution achieved was $\sim 0.05^{\circ}$.

\section{LASING CHARACTERISTICS}

Figure 2(a) shows the pulsed lasing characteristics of the polymer waveguide. The laser had a threshold pump energy of $10 \mathrm{~nJ}$, above which the output energy increased linearly with excitation. The laser beam was emitted along the normal to the waveguide, with approximately equal energies being emitted from each face of the polymer film. At a pump energy of $78 \mathrm{~nJ}$, the total bidirectional output energy was 3.1 $\mathrm{nJ}$, corresponding to a differential quantum efficiency of $5.8 \%$. Figure 2(b) shows the emission spectrum of the structure measured normal to the waveguide when pumped both well below, and above, lasing threshold. The dotted line represents the subthreshold emission, which is dominated by a pair of closely spaced peaks at around $635 \mathrm{~nm}$. The solid line shows the laser spectrum at a pump energy of $49 \mathrm{~nJ}$, approximately five times lasing threshold. We find that lasing occurs at a wavelength of $636 \mathrm{~nm}$, and the linewidth of the laser mode of $0.3 \mathrm{~nm}$ is limited by the resolution of the spectrometer.

As already mentioned, the laser emitted a bidirectional beam along the waveguide normal, typically with an azimuthally polarized, annular transverse mode. At a pump energy of $25 \mathrm{~nJ}$, the half angle divergence of the beam was measured to be $3.8 \pm 0.3 \mathrm{mrad}\left(0.22 \pm 0.02^{\circ}\right)$. For pump energies well above $100 \mathrm{~nJ}$, the transverse laser mode became 

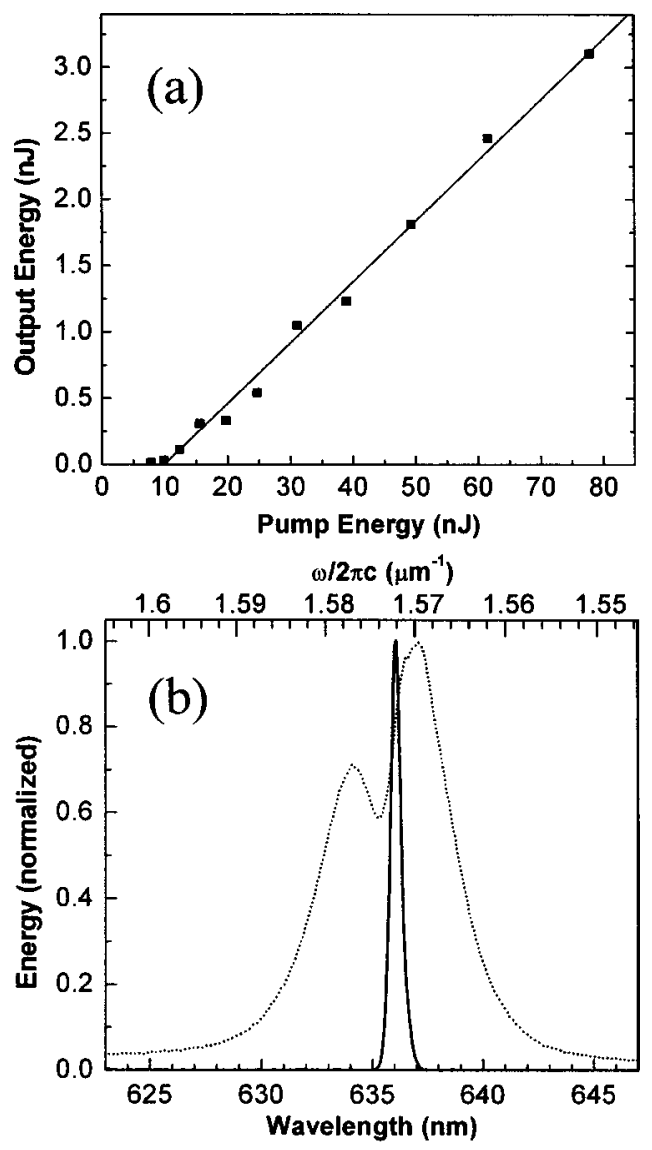

FIG. 2. (a) Output energy from polymer laser as a function of pump energy. (b) Emission spectrum normal to the waveguide plane; dotted line shows spontaneous emission well below lasing threshold, full line shows laser emission when pumped at five times lasing threshold.

significantly more divergent, taking a "cross" profile similar to that observed in previous two-dimensional DFB polymer lasers. ${ }^{14,22}$ The arms of the cross were oriented along the $0^{\circ}$ and $90^{\circ}$ azimuths, with a large half angle divergence of $\sim 400 \mathrm{mrad}\left(\sim 7^{\circ}\right)$ at a pump energy $\sim 1 \mu \mathrm{J}$. Each of the two arms was linearly polarized along its high-divergence axis.

\section{PHOTONIC MODE STRUCTURE OF THE LASER}

\section{A. Background theory}

In this section we discuss the photonic mode structure of the polymer laser, and show how various features of the laser emission arise from the photonic dispersion. First we consider a basic planar air-polymer-silica slab waveguide. The calculated photonic dispersion of this waveguide, plotted in $\omega-k$ space, is shown in Fig. 3 for the spectral range in which MEH-PPV is both transparent and fluoresces. The calculation was based on refractive index data for MEH-PPV determined from angle-dependent reflectivity measurements. ${ }^{27}$ The parameter $k_{\|}$is the component of the photon wave vector parallel to the plane of the guide. The waveguide is cylindrically symmetric and so the dispersion curve is the same for modes with any propagation direction $\phi$. The dotted lines in the figure depict the air and $\mathrm{SiO}_{2}$ light lines, which corre-

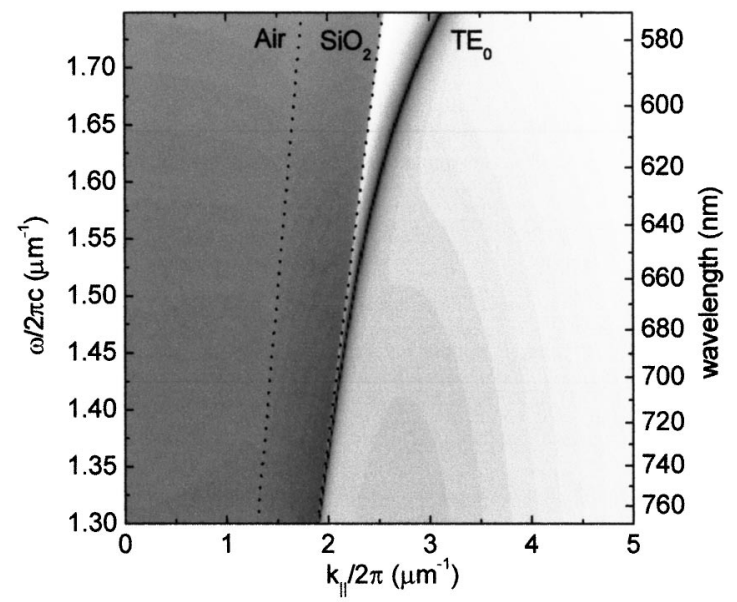

FIG. 3. Calculated mode dispersion of the planar MEH-PPV waveguide.

spond to the critical angles for the air-polymer and polymersilica interfaces, respectively. Bound waveguide modes can only exist to the right of the $\mathrm{SiO}_{2}$ light line, while only leaky modes occur to the left of this line. From the figure it is clear that only the fundamental transverse electric mode $\left(\mathrm{TE}_{0}\right)$ is supported by the guide within the spectral range of interest.

The presence of the bigrating structure breaks the cylindrical symmetry, and modifies this dispersion via Bragg scattering of the $\mathrm{TE}_{0}$ mode by the two orthogonal gratings. The outcome of Bragg scattering on waveguide modes can be usefully visualized in $k$ space. The diagrams in Figs. 4 and 5 provide such a $k$-space illustration, relevant to DFB lasing in our structure. Whereas the curve in Fig. 3 shows how the energy of the unscattered waveguide mode varies with $k_{\|}$(in a planar guide), Figs. 4 and 5 plot the azimuthal dependence of $k_{\|}$at a specific mode energy for the bigrating structure. The dimensions $k_{x}$ and $k_{y}$ define symmetry directions in the plane of the guide, orthogonal to the grating grooves.

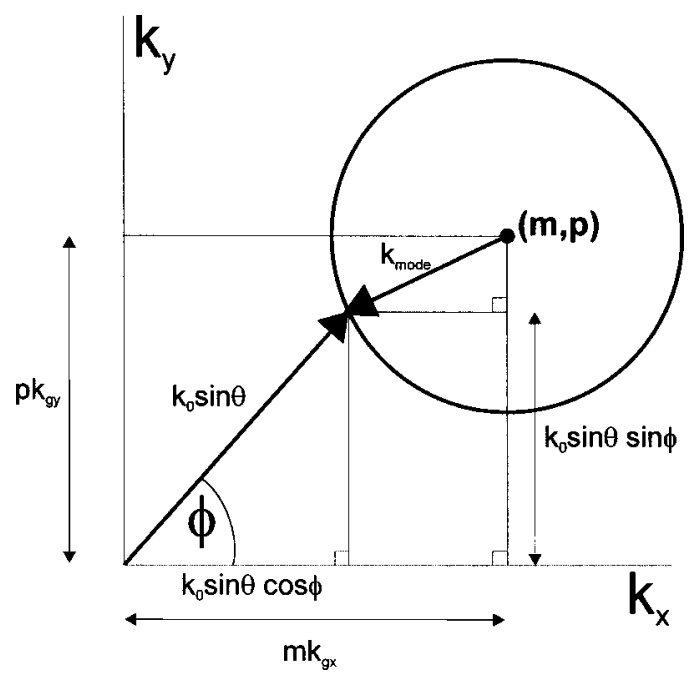

FIG. 4. Construction showing the geometry for general Bragg scattering of waveguide modes. 


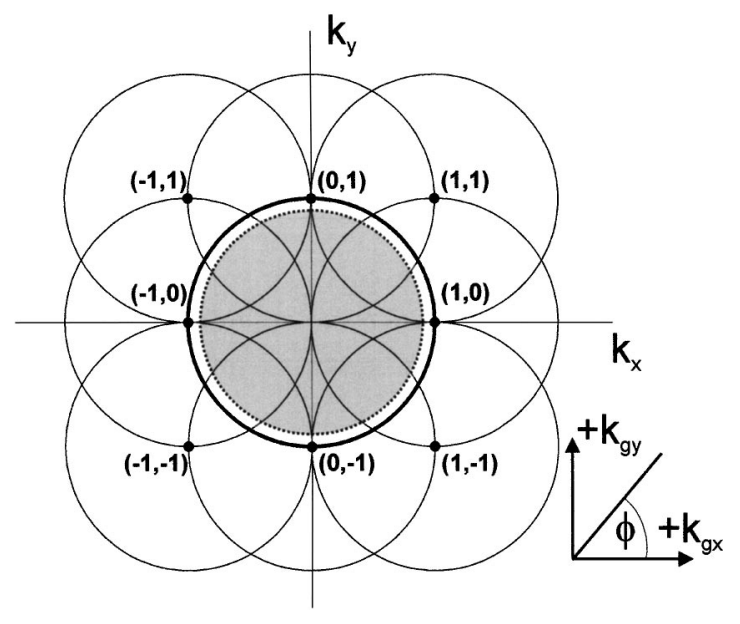

FIG. 5. Bragg scattered mode circles. Bold circle represents unscattered waveguide mode, shaded region represents the range of wave vectors that may couple to radiation.

The wave vector of the unscattered guided mode is represented by a circle (drawn in bold in Fig. 5), centered at the origin of $k$ space, of radius $k_{\text {mode }}=n_{\text {eff }} / \lambda$, where $n_{\text {eff }}$ is the effective waveguide index. The effect of a grating of period $\Lambda$ is to scatter the mode in $k$ space by a value $k_{G}=2 \pi / \Lambda$ along an axis perpendicular to the grating grooves. Figure 4 illustrates the general outcome of multiple scatterings $\left(m k_{G x}+p k_{G y}\right)$ by the two orthogonal grating vectors $k_{G x}$ and $k_{G y}$. The waveguide mode is scattered to a new coordinate in the $k_{x}-k_{y}$ plane with polar coordinates $\left(k_{0} \sin \theta, \phi\right)$. From this geometrical picture we can deduce a simple algebraic expression for the scattering process:

$$
k_{\text {mode }}^{2}=\left(m k_{G x}-k_{0} \sin \theta \cos \phi\right)^{2}+\left(p k_{G y}-k_{0} \sin \theta \sin \phi\right)^{2} .
$$

If the coordinate $\left(k_{0} \sin \theta, \phi\right)$ lies within the (unscattered) $\mathrm{SiO}_{2}$ light cone (the shaded region in Fig. 5), then the mode will be coupled to radiation at a polar angle $\theta$ in the $\phi$ azimuthal plane. If it coincides with another waveguide mode, then energy may be coupled between the two modes.

Figure 5 shows the specific $k$-space diagram for our bigrating laser at a photon energy of $\omega / 2 \pi c=1.57 \mu \mathrm{m}^{-1}$. The bold circle centered on $(0,0)$ represents the unscattered waveguide mode of Fig. 3, and the inner dotted circle represents the $\mathrm{SiO}_{2}$ light line. The two orthogonal gratings generate a 2D array of scattered modes, which are labeled in the figure by the order of the scattering process, i.e., $(m, p)$. These scattered modes couple to radiation where they lie within the shaded circle, and couple to each other at points where they intersect. (This picture is strictly valid only for a vanishingly small level of scattering. Where significant scattering is present, the mode circles can become deformed where gaps form at these intersections. Such effects will be discussed later.)

The magnitude of $k_{\text {mode }}=n_{\text {eff }} / \lambda$, and hence the diameter of the mode circles in Fig. 5, varies with photon energy, changing the nature of couplings between modes. The energy dependence of the scattered modes is shown in Fig. 6, which depicts the dispersion of the $2 \mathrm{D}$ grating structure along the

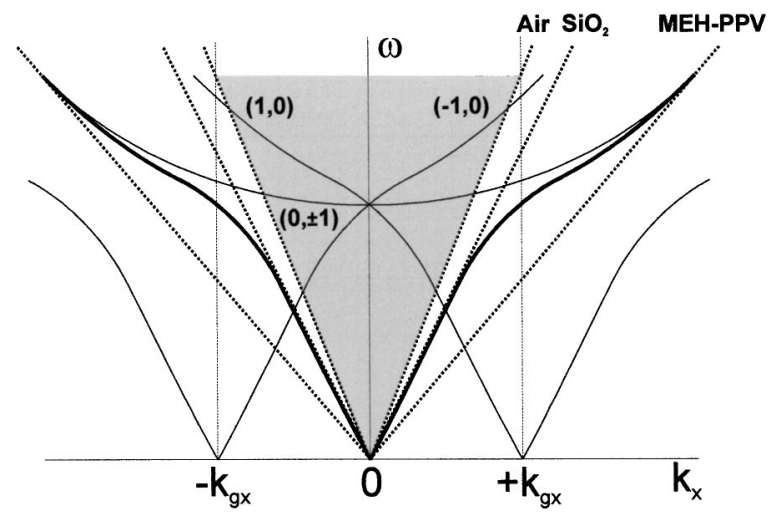

FIG. 6. Schematic of the mode dispersion of the doubly corrugated MEH-PPV waveguide.

$k_{x}$ axis $(\phi=0)$. This figure is constructed by calculating, as a function of photon energy, where the mode circles of Fig. 5 intersect the $k_{x}$ axis. The bold line shows the unscattered mode of Fig. 3, but now there are additional scattered modes, as determined by Eq. (1), corresponding to the circles in Fig. 5 . The modes of the guide are no longer confined between the $\mathrm{SiO}_{2}$ and MEH-PPV light lines, but now also appear within the $\mathrm{SiO}_{2}$ and air light cones where they are readily coupled to radiation by scattering from the periodic microstructure.

At a particular energy, we find that four scattered modes $( \pm 1,0)$ and $(0, \pm 1)$ intersect at $k_{x}=0$. This occurs, in fact, at the energy $\omega / 2 \pi c=1.57 \mu \mathrm{m}^{-1}$ used in Fig. 5. At this energy, counterpropagating modes parallel to $k_{x}$ and $k_{y}$ couple together to provide a $2 \mathrm{D}$ distributed feedback mechanism for lasing. Light from each of these modes will also be scattered out of the guide at an angle $\theta=0$. We may therefore expect to see lasing at this energy, emitted normal to the waveguide. At higher photon energies, we find that the $(0, \pm 1)$ modes also intersect. In this case the wave vectors are not aligned along the same axis, but do have components along the $k_{x}$ axis that counterpropagate. Thus we might also expect to see 1D lasing (albeit with greater losses and therefore with a higher threshold) emitted over a range of polar angles along the $k_{x}\left(\right.$ and $k_{y}$ ) axes.

So the scattering leads to a coupling between the guided modes and free space and, importantly, a coupling between guided modes propagating at different azimuthal angles $\phi$ within the polymer film. The first of these leads to surfaceemitted output coupling from the laser, while the second provides a distributed feedback mechanism. These effects were also present in our study of a 1D DFB polymer laser, ${ }^{10}$ but the presence of the second grating direction in this system significantly increases the number of coupling geometries.

\section{B. Experimental measurement of photonic dispersion}

The simple model of scattered waveguide modes described in the previous section can be readily confirmed experimentally using transmission and photoluminescence measurements of the structure. Such measurements can also reveal more detailed structure of the photonic dispersion, without the need for more complicated theoretical 

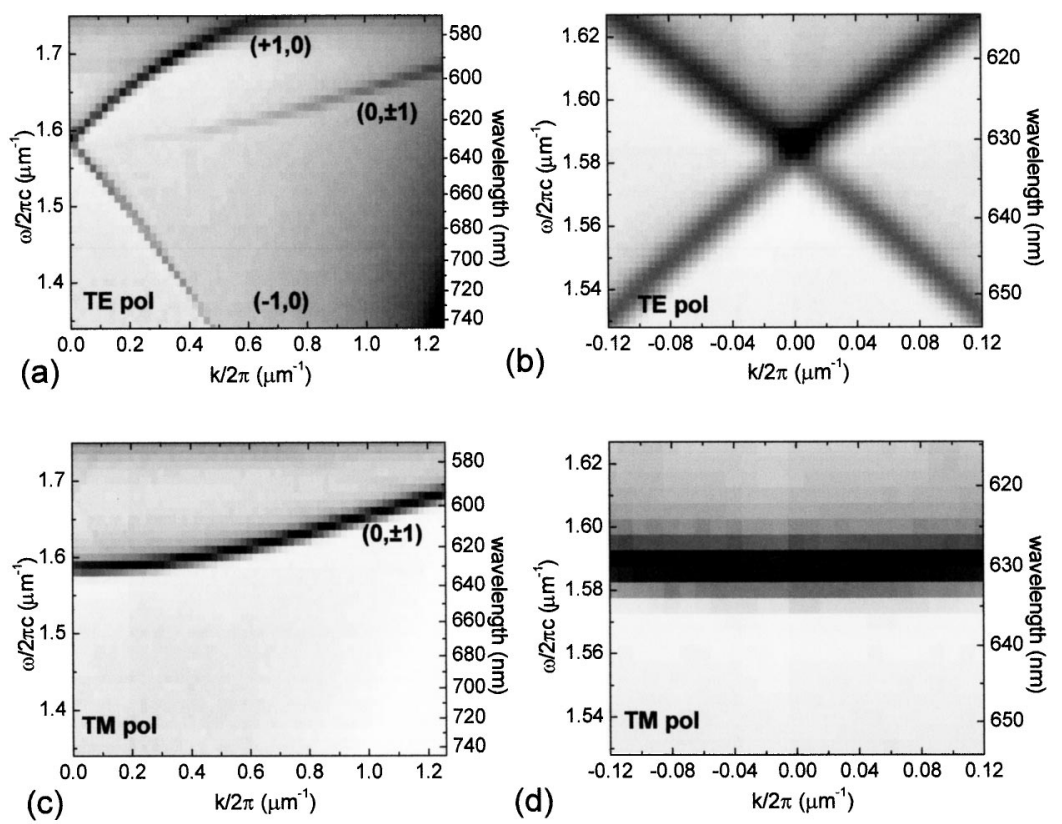

(d)

modeling. ${ }^{10,12}$ Importantly these measurements probe the actual sample under study, and not just a theoretical approximation to the structure. In this section we report direct measurements of the photonic dispersion, over a region corresponding to the shaded area of Fig. 5.

\section{Transmission}

Figure 7 shows the spectral transmission characteristics of the corrugated waveguide. Figures 7(a) and (b) show the transmission of TE polarized light and Figs. 7(c) and (d) the transmission of TM polarized light, all measured in the $0^{\circ}$ azimuthal plane. In Fig. 7, dark regions represent reduced transmission; identical transmission was observed at $\phi$ $=90^{\circ}$ azimuth, and so only the $\phi=0^{\circ}$ data are presented here. We find that the transmission spectra from the $2 \mathrm{D}$ corrugated waveguide is strongly dispersive, similar to that previously observed for $1 \mathrm{D}$ corrugated structures. ${ }^{10,12}$

The transmission spectra are dominated by a pair of minima that diverge with increasing in-plane wave vector. These form two bands of reduced transmission (that we identify from Fig. 6 as $( \pm 1,0)$ scattered modes). Very close to normal incidence, the lower energy band becomes weak (i.e., the reduction in transmission becomes less), while the higher energy band becomes stronger (i.e., a greater reduction in transmission). One other low-transmission band is evident in Fig. 7(a), which we identify as due to $(0, \pm 1)$ scattered modes. This band is flatter than the other two, becomes progressively weaker with reducing in-plane wave vector, and is very faint in Fig. 7(b), where it overlaps with the high energy $( \pm 1,0)$ band.

The corresponding TM polarized transmission at $\phi=0$ is shown in Figs. 7(c) and (d). Only one band of reduced transmission is evident in these figures. This band is similar in form to the $(0, \pm 1)$ band in $7(\mathrm{a})$, though is slightly redshifted, and is fairly constant in strength across all in-plane wave vectors. Before discussing these results further it is instructive to first examine the photoluminescence data. here.
FIG. 7. Transmission dispersion diagrams; (a) and (b) show transmission of TE polarized light, (c) and (d) show transmission of TM polarized light. Dark regions represent minimum transmission.

\section{Photoluminescence}

Figure 8 shows the spectral and angular emission characteristics of the corrugated waveguide when excited well below lasing threshold. Figures 8(a) and (b) show TE, and Figs. $8(\mathrm{c})$ and (d) TM, polarized emission measured in the $\phi$ $=0^{\circ}$ azimuthal plane. In Fig. 8, white regions represent the strongest spontaneous emission. Photoluminescence measurements were made for a range of emission angles (as labeled at the top of each graph). The data are plotted in Fig. 8 on a grid of equally spaced energies and in-plane wave vectors $\left[k_{\|}=(\sin \theta) / \lambda\right]$, to directly relate the spectra to the photonic dispersion of the device. The emission spectra take a very similar form to the transmission data of Fig. 7. The spectral features are all much narrower than the broad intrinsic emission from MEH-PPV that spans the range 580-750 $\mathrm{nm}$. As with the transmission data, identical emission was observed at $90^{\circ}$ azimuth and so only the $0^{\circ}$ data is presented

The TE polarized spectra [Figs. 8(a) and (b)] are dominated by a pair of peaks that split apart with increasing wave vector. These form two bands of emission that roughly overlap with the $( \pm 1,0)$ bands in Figs. $7(\mathrm{a})$ and (b) (the small spectral shift of $\sim 0.012 \mu \mathrm{m}^{-1}(<5 \mathrm{~nm})$ probably arises from sampling slightly different regions of the waveguide in the two experiments). These two emission bands are separated by a shallow dip at $1.57 \mu \mathrm{m}^{-1}$, which can be more clearly seen in the normal incidence photoluminescence curve in Fig. 2(b). The emission peak on the low-energy edge of the dip is significantly narrower and more intense than the peak on the high-energy edge. These two bands resemble the spontaneous emission spectra previously reported from a 1D DFB polymer laser. ${ }^{10}$ The 2D structure exhibits two other bands of emission, labeled $(0, \pm 1)$, whose spectra change more slowly with wave vector. In Fig. 8(a) these bands differ in energy by $0.007-0.017 \mu \mathrm{m}^{-1}$ as the wave vector increases from 0 to $1.25 \mu \mathrm{m}^{-1}$. The band with higher energy overlaps with the $(0, \pm 1)$ transmission band in Fig. 7(a). It becomes faint for $k_{\|}<0.7 \mu \mathrm{m}^{-1}$, and is barely discernible in Fig. 8(b) 


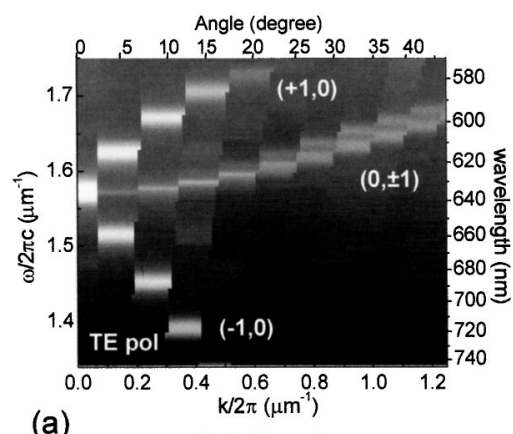

(a)

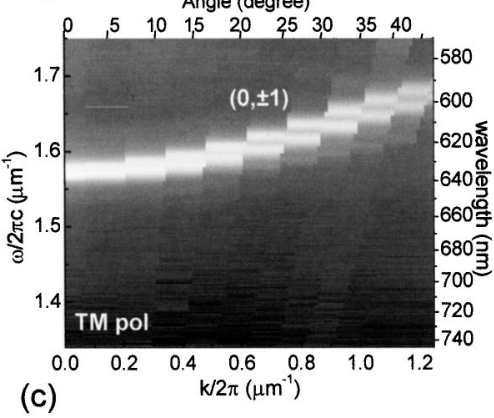

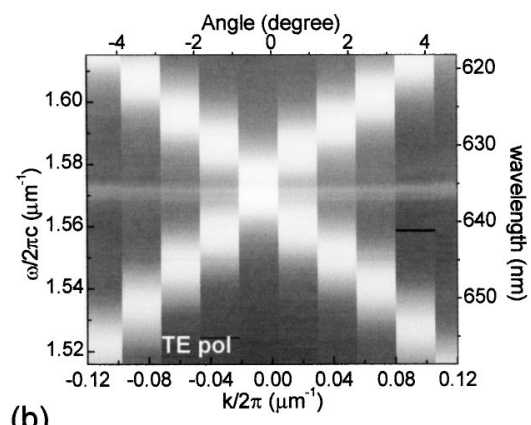

(b)

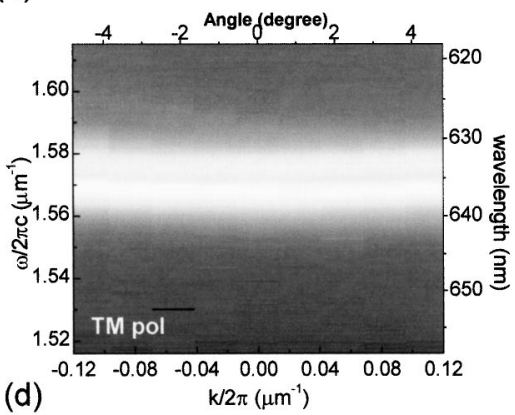

FIG. 8. Photoluminescence dispersion diagrams; (a) and (b) show emission of TE polarized light, (c) and (d) show emission of TM polarized light. Light regions represent maximum emission. where it overlaps with the high-energy $( \pm 1,0)$ mode. Close to the waveguide normal, the lower energy $(0, \pm 1)$ emission band narrows in spectrum and two distinct spectral peaks become just resolvable at 1.572 and $1.570 \mu \mathrm{m}^{-1}$, in Fig. 8(b).

The corresponding TM polarized emission is shown in Figs. 8(c) and (d). This is less complicated than the TE polarized emission and is comprised of only two spectral bands. These bands closely follow the TE polarized $(0, \pm 1)$ emission bands, but are redshifted by between 0.0012 and $0.005 \mu \mathrm{m}^{-1}$ across the $k_{\|}$range. Both TM polarized bands increase in intensity at small $k_{\|}$unlike the TE emission, which becomes significantly fainter.

\section{DISCUSSION}

The strong correspondence between emission and transmission in Figs. 7 and 8 shows that the photonic dispersion of the corrugated structure directly controls the emission properties. In particular, we can readily identify pairs of bands that provide a direct link between the emission from the structure and its transmission. In this section we identify the physical origin of the spectral emission bands, relate their properties to the photonic modes, and discuss the importance of photonic dispersion when considering lasing characteristics.

\section{A. Physical origin of spectral bands}

Referring to Eq. (1), we can straightforwardly identify the physical origin of the transmission and emission bands. These involve the coupling of bound waveguide modes to smaller in-plane wave vectors that lie within the air light cone. In the spectral range of interest, two distinct scattering mechanisms contribute. These are scattering by $\pm k_{G x}$ (i.e., $m= \pm 1, p=0$ ) from one set of grating grooves and scattering by $\pm k_{G y}(m=0, p= \pm 1)$ from the orthogonal grating. In the first mechanism, for each in-plane wave vector, there are two different mode energies that undergo Bragg scattering. The lower energy mode is scattered according to $k_{0} \sin \theta$ $=k_{G x}-k_{\text {mode }}$ while the higher energy mode scatters from the counterpropagating waveguide mode according to $k_{0} \sin \theta$ $=k_{\text {mode }}-k_{G x}$, so leading to the two spectral branches at a given $k_{0} \sin \theta$. The second mechanism $(m=0, p= \pm 1)$ also leads to pairs of bands; the origin of these will be discussed in the next section.

As only the TE guided mode is supported by the structure, the polarization of the light is in general aligned in the plane of the guide, tangentially to the mode circles of Fig. 5. Thus the difference in strength between TE and TM polarized emission can largely be accounted for by the gradient of the circle in Fig. 5, at the point of intersection with the $k_{x}$ azimuthal axis. If the gradient of the circle is perpendicular to the axis, only TE polarized radiation will couple with the waveguide mode; if the gradient is parallel to the axis, only TM polarized radiation will be coupled.

\section{B. Stop bands and mode splitting}

In the previous section, we mentioned that the model employed above was strictly valid only for a vanishingly small level of scattering. In practice, this is not the case, because of the significant depth and anharmonicity of the substrate corrugations, and the high index contrast between the substrate and polymer film. The strong scattering can lead to significant coupling between waveguide modes that propagate in different directions.

This can modify the shape of the photonic dispersion, particularly in regions of the $k$-space diagram (Figs. 4 and 5) where the scattered $k$ vectors intersect. In this section we identify and account for the features in the dispersion data that arise from these couplings.

Close to normal incidence in Fig. 8, there is a dip in the emission [seen clearly in Fig. 2(b)], which corresponds to a 
photonic stop band. Here, second-order Bragg scattering also becomes important; the wavelengths that couple to the guide from normal incidence additionally experience Bragg scattering between counterpropagating modes. These guided modes interfere to set up a standing-wave pattern with a periodicity of $\Lambda / 2$. From symmetry arguments, one finds that the structure supports two such standing waves. ${ }^{28}$ These have nodes either at the maxima and minima, or at the shoulders, of the fundamental spatial component of the grating. Consequently, the standing waves have different energies, and hence different frequencies. A gap opens up in the band structure between these two band edges, within which guided modes always interfere destructively and so cannot propagate in the waveguide. This band-gap feature appears as a reduction in emission in Figs. 2(b) and 8(b) and in coupling in Fig. 7(b) because light can only couple out of or into the waveguide where a mode exists.

There is also a coupling between guided modes propagating at different azimuths. ${ }^{14}$ These occur, for example, at the intersection points of the mode circles in Fig. 5. Importantly, light emitted at $1.57 \mu \mathrm{m}^{-1}$ actually experiences coupling between four scattered modes propagating in different directions ( $m= \pm 1, p=0 ; m=0, p= \pm 1)$, setting up a full $2 \mathrm{D}$ standing-wave pattern of a complex polarization. This polarization effect of the 2D coupling is evident from the presence of TE polarized emission in the $(0, \pm 1)$ bands [Fig. 8(a) and (b)] that was absent from a similar 1D structure. ${ }^{10}$

Feedback also occurs at other intersections between scattered mode circles elsewhere in the photonic dispersion, where partly counterpropagating modes can couple together. By partly counterpropagating we mean that the modes possess equal and opposite wave-vector components when resolved along either one or other of the $k_{G}$-vector directions. The coupling produces a standing-wave field pattern along this $k_{G}$-vector direction, and a traveling wave along the orthogonal direction. This mechanism gives rise to the band splitting of the $(0, \pm 1)$ bands in much the same way as described earlier. Note that in this case the splitting occurs for a range of $k_{\|}$values and not at a single point, since it is generated at the intersection of two scattered mode cones $(0, \pm 1)$, which corresponds to the excitation of partly counterpropagating waves.

Finally in this section, we address the main differences between the emission and transmission spectra. These differences are most prominent in the presence of some bands in emission, which are faint or do not appear at all in transmission. At $k_{\|}=0$ we see that the stronger band edge in emission is the weaker in transmission. These differences occur due to the strength of coupling between the waveguide modes and free space. The transmission measurements give a direct measurement of this coupling strength. Thus the low-energy $( \pm 1,0)$ band edge, for example, is weakly coupled to free space and does not appear in the transmission measurements, while the high-energy $( \pm 1,0)$ edge experiences stronger coupling. The low output coupling of the low-energy band edge leads to a high- $Q$ resonance for light emitted into the waveguide mode from the polymer. Some of this light can be seen in the emission spectra because of weak residual scattering to free space. The high $Q$ of these modes is clear from the emission near $k_{\|}=0$, where the emission becomes very narrow and a secondary longitudinal mode redshifted by $\sim 0.002 \mu \mathrm{m}^{-1}$ appears. The spacing between the modes suggests an effective resonator length of $\sim 200 \mu \mathrm{m}$, roughly equal to the size of the pump excitation area.

The difference in the coupling strengths at the two band edges occurs due to the different spatial distributions of the optical field relative to the physical corrugations. For one band-edge mode (the higher energy mode), the field maxima are vertically displaced from one another, while for the other (lower energy) band-edge mode, the maxima lie in the same horizontal plane relative to the average grating plane. ${ }^{29}$ The fields of the band-edge mode with vertically displaced maxima can easily generate a plane-wave propagating upwards (along the sample normal) and so this mode readily couples to far-field radiation. The fields of the band-edge mode with maxima in the same horizontal plane are not able generate a plane wave emitted normal to the sample (they will cancel in the far field) and so this band-edge mode couples less well to radiation. This difference in the level of output coupling is the origin of the band-edge frequency selection mechanism.

\section{Laser emission}

Having established the photonic dispersion, and its effects on the spontaneous emission from the structure, it is now straightforward to explain the observed lasing phenomena. First, the lasing occurs on the low-energy band edge at 636 $\mathrm{nm}\left(1.572 \mu \mathrm{m}^{-1}\right)$. At this band edge, the emission experiences a strong $2 \mathrm{D}$ coupling of the waveguide modes leading to band flattening and a high density of photon states at the stop-band edges. The 2D coupling creates a complicated polarization pattern in the photonic structure ${ }^{30}$ and leads to the azimuthally polarized surface emission of the laser beam. The difference in coupling strengths of the two band edges to radiation leads to a higher- $Q$ resonance on the longwavelength band edge and hence a significantly lower oscillation threshold. Therefore lasing is first established on the long-wavelength edge.

The 2D nature of the resonance only occurs for a small range of $k_{\|}$in both the $0^{\circ}$ and $90^{\circ}$ azimuthal planes, which leads to the low divergence of the output laser beam. For much higher pump powers, 1D DFB resonances may be independently excited above threshold. These 1D resonances can experience feedback over a wide range of $k_{\|}$(i.e., along the $(0, \pm 1)$ band), which leads to the more divergent emission along each long axis of the observed "cross" pattern.

\section{CONCLUSIONS}

We have measured the emission characteristics and photonic band structure of a 2D DFB laser, made from the conjugated polymer MEH-PPV. We have used knowledge of the photonic dispersion to help explain how the substrate microstructure modifies spontaneous and stimulated emission. The wavelength-scale corrugations provided both a distributed feedback in the plane of the film and coupling between the waveguide mode and free space radiation. We have identified conditions for both 2D and 1D coupling of waveguide 
modes, which leads to a low divergence laser emission at low pump energies, and a much more divergent cross-shaped beam at much higher pump energies. The structure studied showed a 2D stop band around $635 \mathrm{~nm}$, near which lasing occurred on one band edge for excitation energies over 10 nJ. A difference in the output coupling of modes at the two band edges led to the frequency selection; a mechanism clearly illustrated in the photonic dispersion data. The 2D nature of the microstructure also affects the polarization of the emission. This leads to additional TE polarized emission bands not evident in similar 1D structures, and the complex azimuthal polarization of the laser mode.

We have shown how direct measurements of the photonic band structure can be used to explain the spectral and spatial characteristics of laser emission from 2D DFB polymer waveguides. We have uncovered detailed structure in the photonic dispersion, without the need for complicated theo- retical modeling; the dispersion directly relates to the actual sample under study and not just a theoretical approximation to the structure. The combination of measurements used here are very useful for probing the photonic modes of polymer microstructures, and their coupling strengths to radiation. They therefore form a powerful tool for the assessment and understanding of the effects of physical microstructures on both spontaneous and stimulated emission.

\section{ACKNOWLEDGMENTS}

We are grateful to the EPSRC and SHEFC for financial support, and Covion for the supply of the polymer. I.D.W.S. acknowledges the Royal Society University for financial support; G.A.T. acknowledges EPSRC for financial support. The assistance of Jon Wasey in the calculations leading to Fig. 3 is gratefully acknowledged.
*Corresponding author. Email address: w.1.barnes@ex.ac.uk

Corresponding author. Email address: idws@st-andrews.ac.uk

${ }^{1}$ D. Moses, Appl. Phys. Lett. 60, 3215 (1992).

${ }^{2}$ F. Hide, M. A. Diaz-Garcia, B. J. Schwartz, M. R. Andersson, Q. Pei, and A. J. Heeger, Science 273, 1833 (1996).

${ }^{3}$ N. Tessler, G. J. Denton, and R. H. Friend, Nature (London) 382, 695 (1996).

${ }^{4}$ N. Tessler, Adv. Mater. 11, 363 (1999).

${ }^{5}$ M. D. McGehee and A. J. Heeger, Adv. Mater. 12, 1655 (2000).

${ }^{6}$ M. D. McGehee, M. A. Diaz-Garcia, F. Hide, R. Gupta, E. K. Miller, D. Moses, and A. J. Heeger, Appl. Phys. Lett. 72, 1536 (1998).

${ }^{7}$ C. Kallinger, M. Hilmer, A. Haugeneder, M. Perner, W. Spirkl, U. Lemmer, J. Feldmann, U. Scherf, K. Mullen, A. Gombert, and V. Wittwer, Adv. Mater. 10, 920 (1998).

${ }^{8}$ S. Riechel, U. Lemmer, J. Feldmann, T. Benstem, W. Kowalsky, U. Scherf, A. Gombert, and V. Wittwer, Appl. Phys. B: Lasers Opt. B71, 897 (2000).

${ }^{9}$ G. A. Turnbull, T. F. Krauss, W. L. Barnes, and I. D. W. Samuel, Synth. Met. 121, 1759 (2001).

${ }^{10}$ G. A. Turnbull, P. Andrew, W. L. Barnes, and I. D. W. Samuel, Phys. Rev. B 64, 125122 (2001).

${ }^{11}$ G. Kranzelbinder, E. Toussaere, J. Zyss, A. Pogantsch, E. W. J. List, H. Tillmann, and H.-H. Horhold, Appl. Phys. Lett. 80, 716 (2002).

${ }^{12}$ P. Andrew, G. A. Turnbull, I. D. W. Samuel, and W. L. Barnes, Appl. Phys. Lett. 81, 954 (2002).

${ }^{13}$ W. Holzer, A. Penzkofer, T. Pertsch, N. Danz, A. Brauer, E. B. Kley, H. Tillmann, C. Bader, and H.-H. Horhold, Appl. Phys. B: Lasers Opt. B74, 333 (2002).

${ }^{14}$ S. Riechel, C. Kallinger, U. Lemmer, J. Feldmann, K. Gombert, V. Wittwer, and U. Scherf, Appl. Phys. Lett. 77, 2310 (2000).

${ }^{15}$ C. Bauer, H. Giessen, B. Schnabel, E.-B. Kley, C. Schmitt, U.
Scherf, and R. F. Mahrt, Adv. Mater. 13, 1161 (2001).

${ }^{16}$ N. Moll, R. F. Mahrt, C. Bauer, H. Giessen, B. Schnabel, E.-B. Kley, and U. Scherf, Appl. Phys. Lett. 80, 734 (2002).

${ }^{17}$ J. R. Lawrence, G. A. Turnbull, and I. D. W. Samuel, Appl. Phys. Lett. 80, 3036 (2002).

${ }^{18}$ V. G. Kozlov, V. Bulovic, P. E. Burrows, and S. R. Forrest, Nature (London) 389, 362 (1997).

${ }^{19}$ M. Meier, A. Dodabalapur, J. A. Rodgers, R. E. Slusher, A. Mekis, A. Timko, C. A. Murray, R. Ruel, and O. Nalamasu, J. Appl. Phys. 86, 3502 (1999).

${ }^{20}$ V. G. Kozlov, G. Parthasarathy, P. E. Burrows, V. B. Khalfin, J. Wang, S. Y. Chou, and S. R. Forrest, IEEE J. Quantum Electron. 36, 18 (2000).

${ }^{21}$ M. Nagawa, M. Ichikawa, T. Koyama, H. Shirai, Y. Taniguchi, A. Hongo, S. Tsuji, and Y. Nakano, Appl. Phys. Lett. 77, 2641 (2000).

${ }^{22}$ S. Riechel, U. Lemmer, J. Feldmann, S. Berleb, A. G. Muckl, W. Brutting, A. Gombert, and V. Wittwer, Opt. Lett. 26, 593 (2001).

${ }^{23}$ M. Meier, A. Mekis, A. Dodabalapur, A. Timko, R. E. Slusher, J. D. Joannopoulos, and O. Nalamasu, Appl. Phys. Lett. 74, 7 (1999).

${ }^{24}$ A. Mekis, M. Meier, A. Dodabalapur, R. E. Slusher, and J. D. Joannopoulos, Appl. Phys. A: Mater. Sci. Process. A69, 111 (1999).

${ }^{25}$ M. Notomi, H. Suzuki, and T. Tamamura, Appl. Phys. Lett. 78, 1325 (2001).

${ }^{26}$ M. G. Salt and W. L. Barnes, Opt. Commun. 166, 151 (1999).

${ }^{27}$ A. Boudrioua, P. A. Hobson, B. Matterson, I. D. W. Samuel, and W. L. Barnes, Synth. Met. 111, 545 (2000).

${ }^{28}$ W. L. Barnes, T. W. Priest, S. C. Kitson, and J. R. Sambles, Phys. Rev. B 54, 6227 (1996).

${ }^{29} \mathrm{P}$. Andrew and W. L. Barnes (unpublished).

${ }^{30} \mathrm{~S}$. Noda, M. Yokoyama, M. Imada, A. Chutinan, and M. Mochizuki, Science 293, 1123 (2001). 